\title{
Afferent Neural Branching at Human Acupuncture Points: Do Needles Stimulate or Inhibit?
}

\author{
Morry Silberstein, $\mathrm{MD},{ }^{1}$ Katharine Adcroft, $\mathrm{MSc}^{1}$, Aston Wan, $\mathrm{MB},{ }^{2}$ and Masimilliano Massi, $\mathrm{PhD}^{1}$
}

\begin{abstract}
Background: Acupuncture has previously been considered to be stimulatory to the nervous system; however, the specific mechanism for this remains unknown, with the few published studies of acupuncture-point histology showing reduced numbers of nerves and neural receptors at acupuncture-point sites.

Objective: This study was undertaken to visualize the neuroanatomic features of acupuncture points in humans. Materials and Methods: Light microscopy was performed on silver-stained sections of a human cadaver at $\mathrm{P}$ 6, and confocal microscopy was performed on PgP9.5 and $\mathrm{P}_{2} \mathrm{X}_{3}$ immunostained sections of 2 points (GB 20 and SP 6) from a live human volunteer.

Results: At each point, but not at control sites, a single nerve bundle extending to the dermal-epidermal junction was identified where it branched into two parts, with each branch running perpendicularly, parallel to the dermal-epidermal junction.

Conclusions: Acupuncture may incise afferent unmyelinated axonal branch points, disrupting both neural transmission to the spinal cord and crosstalk along meridians, while simultaneously stimulating larger, myelinated afferents, thus explaining both the immediate and long-lasting effects of acupuncture.
\end{abstract}

Key Words: Acupuncture, Unmyelinated Afferents, C-Fibers, Meridians

\section{INTRODUCTION}

A Lthough it is CONTroversial, most published evidence suggests that acupuncture produces specific clinical effects, including, but not limited to, pain modulation. ${ }^{1}$ Historically, acupuncture involved insertion of needles into specific body sites (i.e., acupuncture points), each of which was believed to be associated with a specific clinical effect. Although some clinical trials have failed to show significant differences between sham and genuine (or verum) acupuncture, recent work suggests that needling of acupuncture points results in specific cerebral responses and that such needling results in better outcomes than sham-point puncturing. ${ }^{2}$

Acupuncture has uniformly been considered to be stimulatory, although the specific substrate being stimulated has yet to be elucidated. ${ }^{1}$ Despite considerable evidence that the effects of acupuncture are mediated through the nervous system, the only published study of nerve immunohistochemistry at acupuncture points-performed upon human cadaveric material-showed reduced numbers of nerves at acupuncture-point sites, compared to control sites, ${ }^{3}$ while an earlier study in guinea pigs suggested that acupuncturepoint sites had fewer cutaneous receptors, compared to other parts of the skin. ${ }^{4}$ Noting that there is peripheral crosstalk among unmyelinated afferents, it was previously suggested that acupuncture may disrupt this crosstalk by incising these afferents, ${ }^{5}$ thus producing an inhibitory effect. Hence, the current study was undertaken to elucidate the specific neuroanatomical features, if any, of acupuncture points. Noting that cutaneous unmyelinated afferents express $\mathrm{P}_{2} \mathrm{X}_{3}$ receptors and the recent suggestion by Burnstock that acupuncture's effect may be mediated by adenosine triphosphate

\footnotetext{
${ }^{1}$ Department of Chemistry, Curtin University, Perth, Western Australia, Australia.

${ }^{2}$ Department of Pain Management, St. Vincent's Hospital Melbourne, Victoria, Fitzroy, Victoria, Australia.
} 
(ATP) activation of purinergic receptors at acupuncture points, ${ }^{6}$ another goal was to identify purinergic nerves at acupuncture points.

To determine whether acupuncture points have unique neuroanatomical features, an initial examination was made of silver-stained skin biopsies from a preserved human cadaver and then PgP9.5 and $\mathrm{P}_{2} \mathrm{X}_{3}$ immunohistochemistry testing was performed on on excisional biopsies of two acupuncture-point areas from a living human subject (author, M.S.), comparing these with controls sections from the same person.

\section{MATERIALS AND METHODS}

\section{Ethics Statement}

This study was approved by the institution's research ethics committee (Curtin University Human Ethics approval No. SMEC-60-09).

\section{Human Cadaver Skin}

Skin from the volar aspects of both forearms was obtained from a 78 -year-old female cadaver, fixed whole in $10 \%$ formaldehyde solution 3 years prior. Following localization, the left P 6 acupuncture point was excised as a 2-cm block, and a similar-sized section was obtained from her right forearm from a location $2 \mathrm{~cm}$ proximal and lateral to the right $\mathrm{P}$ 6. The long axis of the Pericardium meridian was labeled at the periphery of each block. Blocks were embedded in paraffin according to conventional procedures and serial sagittal $20-\mu \mathrm{m}$ sections were taken from each block parallel to the meridian. The mounted sections were impregnated with silver and scrutinized systematically.

\section{Live Human Skin}

Two right-sided acupuncture points (GB 20 and SP 6) were excised without regional anesthesia (to minimize neural damage) from a live human volunteer (author M.S.) by a physician who was trained in acupuncture (author A.W.) using a 3-mm punch biopsy needle (Kai Medical, ${ }^{\circledR}$ Japan), and associated meridians were labeled. Tissue was fixed in $2 \%$ paraformaldehyde with picric acid and embedded in paraffin, and serial sagittal $20-\mu \mathrm{m}$ sections were taken from each block parallel to the meridian. Sections underwent double-stain PgP9.5 and $\mathrm{P}_{2} \mathrm{X}_{3}$ immunocytochemistry using accepted techniques. Mounted sections were examined with confocal scanning laser microscopy (Nikon upright Eclipse 90i). Results of this microscopy were compared with paired control biopsies from the contralateral body side of the same person at some distance to known acupuncture points, sectioned parallel to the spine (upper thoracic) or tibia (left calf). Both acupuncture point sites had prominent pulsatile (small) arterial bleeding during the biopsies, indicating a neurovascular base, in contrast what occurred at the control biopsy sites.

\section{RESULTS}

Sections parallel to the Pericardium meridian, from the human cadaver's P 6 acupuncture point had a solitary, thin, and moderately argyrophilic structure, extending vertically through the superficial dermis and bifurcating at the dermalepidermal junction (Fig. 1). No similar nervelike structures

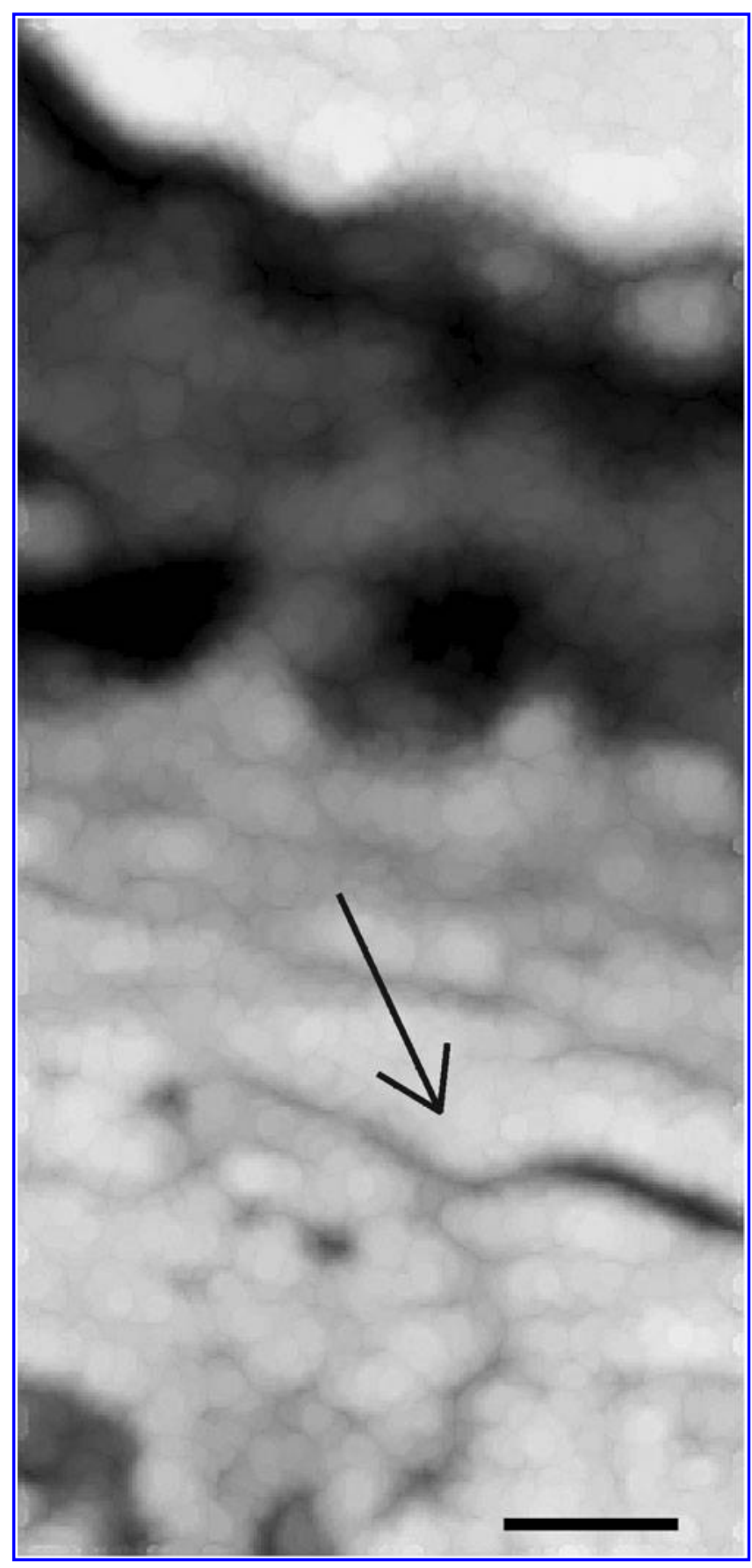

FIG. 1. Neural branch point at P 6 (Neiguan) from silver-stained human cadaver skin. "T"'-shaped arygyrophilic structure (see arrow) in superficial dermis from left hand $\mathrm{P} 6$ of a human cadaver at $10 \times$ magnification. 
were visible elsewhere in the P 6 sections or on contralateral control skin sections.

In immunostained live human sections parallel to the Spleen and Gallbladder meridians, respectively, the following specific observations were made at both acupuncture points only (Fig. 2):

(1) Large nerve trunks at the base of each point

(2) Prominent fibrous bands, extending vertically toward the dermal-epidermal junction, where they bifurcated parallel to associated meridians

(3) A solitary PgP9.5-positive neural bundle at each acupuncture point, extending to the dermal-epidermal junction, where it branched into two parts, with each new branch running perpendicularly, parallel to the dermal-epidermal junction

(4) Occasional $P_{2} X_{3}$-positive axons within this neural bundle.

\section{DISCUSSION}

While the exact mechanism by which acupuncture produces its clinical effect is unknown, most published evi- dence suggests that this involves afferent nerves at acupuncture points. ${ }^{7}$ In contrast, the only previous published descriptions of point histology (from a human cadaver $^{8}$ and a guinea pig, ${ }^{9}$ respectively) have shown reduced numbers of nerves ${ }^{8}$ and receptors. ${ }^{9}$ The current study sought to resolve this paradox by examining, for the first time, tissue from a live human, without regional anesthesia, to minimize any distortion. While this study was limited, being only based upon only two acupuncture point biopsies from a live human, as well as a solitary cadaver acupuncture point, the results raise an intriguing question: If acupuncture points are sites of neural branching, could acupuncture's effect be the result of needle disruption of this branch point?

Unlike initial fast-onset pain, chronic pain-for which acupuncture has also shown significant clinical effects-is mediated by unmyelinated C-fibers ${ }^{1}$ and, consistent with Burnstock's suggestion that acupuncture's effect involves purinergic $\mathrm{P}_{2} \mathrm{X}_{3}$ receptors, ${ }^{6}$ the current study found occasional $\mathrm{P}_{2} \mathrm{X}_{3}$ positive axons within the acupuncture-point nerve bundles. Both stimulation of peripheral $\mathrm{C}$-fibers ${ }^{7}$ and peripheral nociceptive stimuli ${ }^{8}$ have been shown to evoke periaqueductal gray excitatory activity. Conversely, acupuncture, to genuine, but not sham, points, results in a

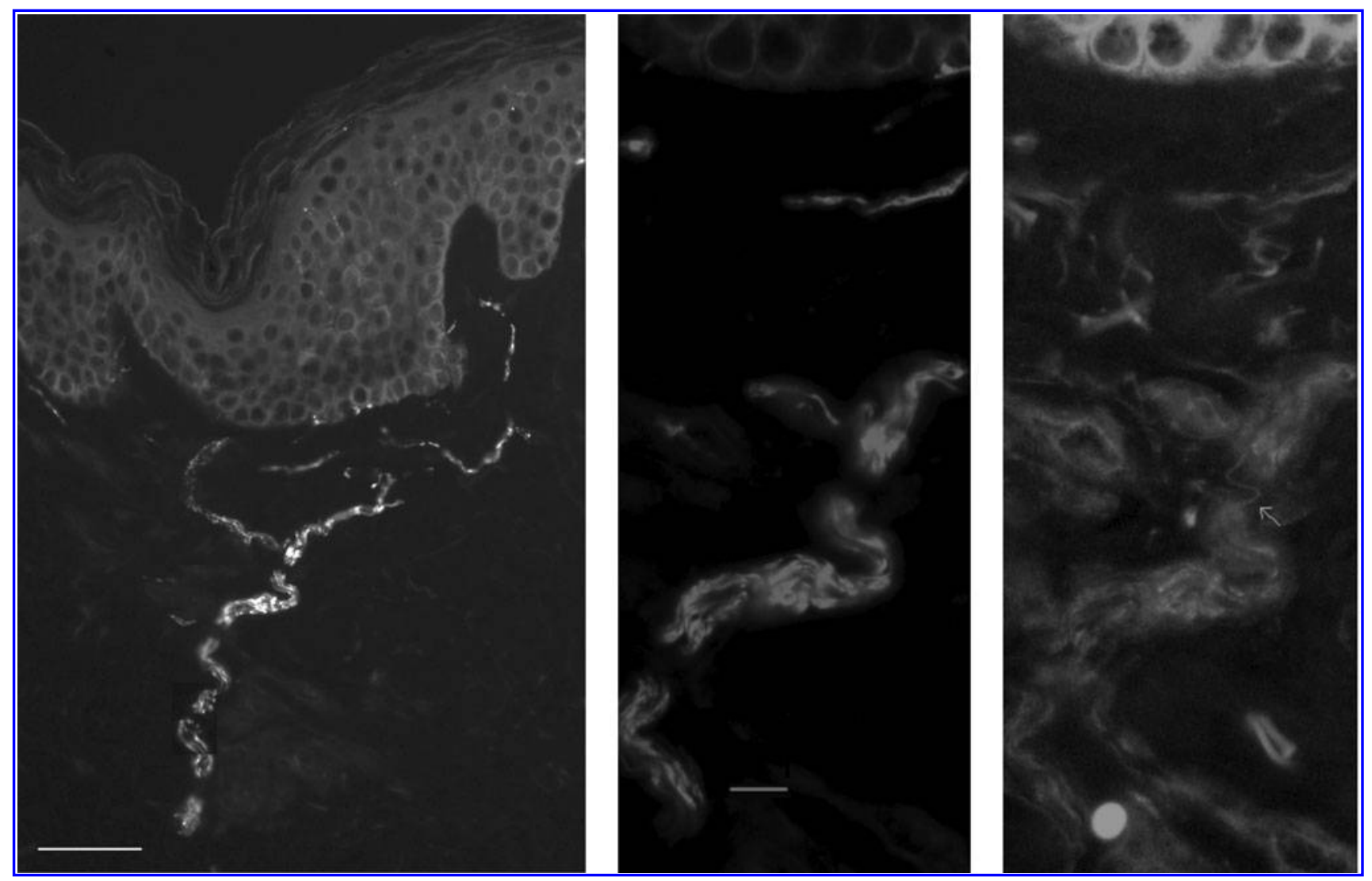

FIG. 2. PgP9.5 and $\mathrm{P}_{2} \mathrm{X}_{3}$ immunostaining of right GB 20 from live human subject. Left: $20 \times$ magnification composite image of two sequential 10- $\mu \mathrm{m}$ sections from GB 20 reveals branching axon bundle. Paired PgP9.5 (center) and $\mathrm{P}_{2} \mathrm{X}_{3}$ (right) $40 \times$ magnification sections of bundle with $\mathrm{P}_{2} \mathrm{X}_{3}$ positive axon (see arrow). Scale bars: $100 \mu \mathrm{m}$ (left); $20 \mu \mathrm{m}$ (center and right). 
significant reduction in periaqueductal gray activity, ${ }^{9}$ and, as described by Theysohn and colleagues at the 96th Annual Meeting Radiologic Society of North America, in Chicago, IL, in 2010, acupuncture reduces cortical activation induced by peripheral painful stimuli, as shown on functional magnetic resonance imaging. ${ }^{10}$ Needle disruption of branching unmyelinated afferents at acupuncture points might also, in addition to reducing nociceptive neurotransmission centrally, interrupt longitudinal C-fiber crosstalk, ${ }^{5}$ explaining, for the first time, the concept of acupuncture meridians. At each of the acupuncture points examined in the current studies, neural branches ran perpendicularly in the plane of the section, which had been previously determined to be parallel to the point's associated meridian. Acupuncture interruption of longitudinal connections between acupuncture points, either via paracrine mechanisms or Merkel cells, 5 would explain the "long-distance" effects of acupuncture. Finally, the superficial location of neural branch sites at acupuncture points would make them amenable to laser acupuncture, which has produced clinical effects that are comparable to needle-based treatment. ${ }^{11}$

However, if acupuncture were purely disruptive, this would conflict with previous findings of abolition of its effect by injection of a local anaesthetic into an acupuncture point prior to acupuncture. ${ }^{1}$ While unmyelinated (purinergic) axons were certainly identified at acupuncture points, neurophysiological studies have confirmed the additional presence of larger nerves, including $\mathrm{A} \beta$ fibers. ${ }^{1}$ Could acupuncture stimulation of these nerves have an inhibitory effect on nociception? Langevin and colleagues have shown that the unique fibrous structure of APs results in accentuated transmission of forces during needling, which would, likely, be transmitted by $\mathrm{A} \beta$ fibers. ${ }^{12}$ There is recent evidence indicating that a subset of inhibitory lamina I dorsal-horn nociceptive neurons receive $\mathrm{A} \beta$ fiber input, ${ }^{13}$ and their stimulation, as might occur during acupuncture, would cause nociceptive inhibition or "closing of the gate," in line with classical theory. Hence, acupuncture could have a dual effect, combining $\mathrm{A} \beta$ stimulation with $\mathrm{C}$-fiber disruption, each resulting in nociceptive inhibition and also explaining both the immediate and long-lasting effects of acupuncture.

\section{CONCLUSIONS}

Branching afferent nerve bundles were identified in this limited study of human acupuncture points, a finding that was not present at contralateral control skin areas. The major limitation of this study is the paucity of data resulting from the difficulty in obtaining volunteers who were willing to undergo point excision. Nevertheless, it is suggested that that needle acupuncture produces an inhibitory effect through a dual mechanism: (1) incising branch points of unmyelinated afferent axons, which have both central connections via the spinal cord and peripheral connections to axons originating from adjacent APs along a meridian; and (2) stimulation of myelinated mechanosensitive axons that produce an inhibitory nociceptive effect on dorsal-horn neurons. This mechanism would-if confirmed by neurophysiological studies-for the first time, explain both the immediate and long-lasting effects of acupuncture.

\section{ACKNOWLEDGMENTS}

The authors acknowledge Michal Schneider-Kolsky, $\mathrm{PhD}$, and Claude Bernard, $\mathrm{PhD}$, of Monash University, Melbourne, Australia, for assistance with staining cadaver material; the University of Western Australia's Cell Central Histology Unit, Perth, Australia, for assistance with immunostaining; Marshall Devor, $\mathrm{PhD}$, and Shi-Hong Zhang, $\mathrm{PhD}$, for advice on the manuscript, and Curtin University's Internal Grant Scheme for funding support.

\section{DISCLOSURE STATEMENT}

The authors confirm that they have no competing financial interests or any other conflict of interest in relation to the work herein described.

\section{REFERENCES}

1. Wang S-M, Kain ZN, White P. Acupuncture analgesia: I. the scientific basis. Anesth Analg. 2008;106(2):602-610.

2. Kong J, Kaptchuk TJ, Polich G, Kirsch I, et al. Expectancy and treatment interactions: a dissociation between acupuncture analgesia and expectancy evoked placebo analgesia. Neuroimage. 2009;45(3):940-949.

3. Wick F, Wick N, Wick MC. Morphological analysis of human acupuncture points through immunohistochemistry. $\underline{A m ~ J}$ Phys Med Rehabil. 2007;86(1):7-11.

4. Monteiro-Riviere NA, Hwang YC, Stromberg MW. Light microscopic morphology of low resistance skin points in the guinea pig. Am J Chin Med. 1981;9(2):155-163.

5. Silberstein M. The cutaneous intrinsic visceral afferent nervous system: a new model for acupuncture analgesia. $\underline{J \text { Theor }}$ Biol. 2009;261(4):637-642.

6. Burnstock G. Acupuncture: a novel hypothesis for the involvement of purinergic signalling. Med Hypotheses. 2009; 73(4):470-472.

7. Collins WF, Randt CT. Midbrain evoked responses relating to peripheral unmyelinated or " $\mathrm{C}$ " fibers in cat. J Neurophysiol. 1960;23:47-53.

8. Hsieh JC, Ståhle-Bäckdahl M, Hägermark O, Stone-Elander $\mathrm{S}$, Rosenquist $\mathrm{G}$, Ingvar M. Traumatic nociceptive pain activates the hypothalamus and the periaqueductal gray: a positron emission tomography study. Pain. 1996;64(2): 303-314.

9. Zyloney CE, Jensen K, Polich G, Loiotile RE, et al. Imaging the functional connectivity of the periaqueductal gray during 
genuine and sham electroacupuncture treatment. Mol Pain 2010;6:80-86.

10. Theyson N, Choi K, Gizewsla ER, Dobos GJ, Forsting M, Musial F. Influence of acupuncture on pain modulation during electrical stimulation: An fMRI study [presentation]. Radiological Society of North America 2010 Scientific Assembly and Annual Meeting, Chicago, IL, November 28-December 3, 2010

11. Dorsher PT. Clinical equivalence of laser needle to metal acupuncture needle in treating musculoskeletal pain: a pilot study. Med Acupunct. 2010;22(1):11-17.

12. Langevin HM, Churchill DL, Cipolla MJ. Mechanical signaling through connective tissue: A mechanism for the therapeutic effect of acupuncture. FASEB J. 2001;15(12):22752282.
13. Takazawa T, McDermott AB. Synaptic pathways and inhibitory gates in the spinal cord dorsal horn. Ann N Y Acad Sci. 2010;1198:153-158.

Address correspondence to: Morry Silberstein MD

Department of Chemistry Curtin University G.P.O. Box U1987

Perth 6845, Western Australia

Australia

E-mail: m.silberstein@curtin.edu.au 